\title{
BMJ Open Study protocol for a randomised controlled multicentre study: the Foraminotomy ACDF Cost-Effectiveness Trial (FACET) in patients with cervical radiculopathy
}

A E H Broekema, ${ }^{1} \mathrm{~J}$ M A Kuijlen, ${ }^{1,2}$ G A T Lesman-Leegte, ${ }^{3}$ R H M A Bartels, ${ }^{4}$ A D I van Asselt,, ${ }^{3,5}$ P C A J Vroomen, ${ }^{6} \mathrm{~J}$ M C van Dijk, ${ }^{1}$ M F Reneman, ${ }^{7}$ R Soer, ${ }^{2,8}$ R J M Groen, ${ }^{1}$ on behalf of the FACET study group investigators

To cite: Broekema AEH, Kuijlen JMA, LesmanLeegte GAT, et al. Study protocol for a randomised controlled multicentre study: the Foraminotomy ACDF Cost-Effectiveness Trial (FACET) in patients with cervical radiculopathy. BMJ Open 2017;7:e012829. doi:10.1136/bmjopen-2016012829

- Prepublication history for this paper is available online. To view these files please visit the journal online (http://dx.doi.org/10.1136/ bmjopen-2016-012829).

JMAK and RS have initiated and designed the FACET study.

Received 26 May 2016 Revised 19 September 2016 Accepted 14 October 2016

CrossMark

For numbered affiliations see end of article.

Correspondence to

JMA Kuijlen;

j.m.a.kuijlen@umcg.nl

\section{ABSTRACT}

Introduction: Cervical radiculopathy due to discogenic or spondylotic stenosis of the neuroforamen can be surgically treated by an anterior discectomy with fusion (ACDF) or a posterior foraminotomy (FOR). Most surgeons prefer $A C D F$, although there are indications that FOR is as effective as ACDF, has a lower complication rate and is less expensive. A head-tohead comparison of the 2 surgical techniques in a randomised controlled trial has not yet been performed. The study objectives of the Foraminotomy ACDF Cost-Effectiveness Trial (FACET) study are to compare clinical outcomes, complication rates and cost-effectiveness of FOR to ACDF.

Methods and analysis: The FACET study is a prospective randomised controlled trial conducted in 7 medical centres in the Netherlands. The follow-up period is 2 years. The main inclusion criterion is a radiculopathy of the $\mathrm{C4}, \mathrm{C5}, \mathrm{C} 6$ or $\mathrm{C} 7$ nerve root, due to a single-level isolated cervical foraminal stenosis caused by a soft disc and/or osteophytic component, requiring operative decompression. A sample size of 308 patients is required to test the hypothesis of clinical non-inferiority of FOR versus ACDF. Primary outcomes are: 'operative success', the measured decrease in radiculopathy assessed by the visual analogue scale and 'patient success', assessed by the modified Odom's criteria. Secondary outcomes are: Work Ability Index (single-item WAI), quality of life (EuroQol 5 Dimensions 5 level Survey, EQ-5D-5L), Neck Disability Index (NDI) and complications. An economic evaluation will assess cost-effectiveness. In addition, a budget impact analysis will be performed.

Ethics and dissemination: Ethical approval was obtained from the Institutional Ethics Committee of the University Medical Center Groningen. Results of this study will be disseminated through national and international papers. The participants and relevant patient support groups will be informed about the results of the study.

Trial registration number: NTR5536, pre-results.

\section{Strengths and limitations of this study}

- The Foraminotomy ACDF Cost-Effectiveness Trial (FACET) study is the first randomised controlled trial investigating anterior discectomy with fusion versus foraminotomy for cervical foraminal pathology.

- Methodological strengths include an appropriate sample size based on a non-inferiority design, seven participating medical centres and an independent institute facilitating the randomisation system and monitoring activities.

- The study is performed with care as usual; therefore, the final results of this study could be generalisable to usual clinical practice.

- With respect to the evaluation of spinal fusion and instrumentation failure, the relatively short follow-up period of 2 years could be a limitation of this study.

- In this study, it is not feasible to blind the participant and the surgeon to the allocated treatment.

\section{INTRODUCTION}

Background and rationale

Cervical radiculopathy has a serious impact on a patient's quality of life (QoL). Many patients with a cervical radiculopathy are in their productive phase of life and are part of their community's labour force. Therefore, temporary disability due to the radiculopathy may result in a major loss of productivity.

A cervical radicular syndrome (CRS) constitutes pain, paresis and/or paraesthesia in the distribution area of the corresponding nerve root. It can be caused by nerve root compression due to soft disc protrusion or by a spondylotic stenosis of the neuroforamen. The annual incidence of a CRS is 
estimated at 0.8 per 1000 inhabitants. ${ }^{1}$ The initial treatment is primarily conservative. The revised Dutch CRS guideline (2010) states that a CRS existing for longer than 2 months, and not responding to conservative treatment in that time course, can be considered an indication for surgical treatment. ${ }^{2}$

Various surgical approaches for the treatment of cervical radiculopathy have been described. Spurling and Scoville $^{3}$ reported the successful removal of the cervical disc with a hemilaminectomy in 1944. In the following years, the techniques further evolved into a keyhole foraminotomy (FOR) being popularised by Frykholm. ${ }^{4}$ Cloward, ${ }^{5}$ Smith and Robinson ${ }^{6}$ developed the anterior approach for cervical discectomy in 1958. Since then, anterior cervical discectomy with fusion (ACDF) has become very popular and became the favourite treatment option in the majority of neurosurgeons and orthopaedic surgeons for cervical degenerative disc diseases with stenosis of the central spinal canal. ${ }^{78}$

For lateral pathologies, such as the foraminal soft disc protrusion or spondylotic stenosis of the neuroforamen, there is still a controversy regarding the best surgical approach. Both the anterior and posterior techniques are well established, but the decision to choose one technique above the other is often based on the surgeon's preference and not on scientific evidence..$^{9-11}$

The majority of surgeons seem to favour the anterior approach. This preference is probably based on the fact that the anterior approach is also used for other cervical disorders, causing the surgeon to be more familiar with this operative route. Furthermore, the assumption that the posterior approach elicits more postoperative neck pain makes this technique less appealing for surgeons. ${ }^{12}$

However, there are also several advantages of the posterior approach in patients with a foraminal soft disc protrusion or spondylotic stenosis.

Four of these advantages are:

1. Clinical outcome after a posterior cervical FOR appears non-inferior to ACDF. There are conflicting results, derived from low-quality, mainly retrospective studies. $^{9-11} 13$ In a large study of Dohrmann and Hsieh, ${ }^{14} 6000$ patients were analysed retrospectively from the existing literature. A good/excellent result (relief of symptoms) was scored in $80 \%$ of the patients after an anterior decompression versus $94 \%$ of the patients after a posterior decompression. This difference was statistically significant $(\mathrm{p}<0.05) .{ }^{14}$ On the other hand, Korinth et $a l^{11}$ found, in a retrospective study of 292 patients, a statistically significant difference in success rate (Odom's criteria I and II) in favour of ACDF with polymethylmethacrylate (PMMA) stabilisation compared with FOR. Furthermore, Wirth and colleagues conducted a prospective trial comparing (1) anterior cervical discectomy without fusion (ACD), (2) ACDF and (3) FOR. The study did not show a significant difference in outcome between the three procedures (pain improvement in $100 \%$ of the patients after ACDF or FOR, 92\% after ACD). However, the number of patients included in the study was very low $(\mathrm{n}=72) .{ }^{10}$

2. Morbidity data are in favour of FOR. The lower complication rate for FOR can be explained by the difference in anatomical structures that are involved in the two approaches. Owing to the proximity of vulnerable and vital structures such as the carotid artery, vagal nerve, trachea, oesophagus and the recurrent laryngeal nerve, ACDF has a higher complication rate. The reported numbers of complications in the literature vary between $6.5 \%$ and $19.3 \%$ for the ACDF technique, and between $1.8 \%$ and $3.9 \%$ in the case of FOR. $^{13} 15-19$

3. Direct costs of FOR seem to be lower. The explanation for this is most likely the high costs of implants used in ACDF. Mansfield et $a l^{18}$ reported an average difference of $\$ 3872$ between ACDF and FOR, with $\$ 3143$ of this difference contributable to costs in the operating room (including materials). Tumialán et $a l^{20}$ found a difference of $\$ 6508$ between FOR and ACDF with plating. Approximately half of these extra costs were caused by the implants used.

4. Indirect costs of FOR seem lower, which may be related to a faster rehabilitation and an earlier resumption of work. However, this assumption is based on two retrospective studies comparing the cost-effectiveness of FOR to ACDF with plating. A comparison to ACDF without plating has not yet been made. ${ }^{20} 21$

In summary, a high-quality study comparing the effectiveness of FOR to ACDF has not yet been performed. Patients, the medical community and financial stakeholders would benefit from a study that puts both procedures to the test. The results of this study could be of use for the update/revision of the (inter) national guidelines for the treatment of a CRS.

\section{Aims and hypotheses}

The Foraminotomy ACDF Cost-Effectiveness Trial (FACET) study is designed as a high-quality study to analyse the cost-effectiveness of the FOR technique compared with ACDF in patients with a cervical monoradiculopathy caused by a discogenic and/or osteophytic foraminal stenosis.

The primary hypotheses are:

- The effectiveness of the FOR technique is noninferior compared with the ACDF technique.

- The FOR technique is cost-effective compared with the ACDF technique.

The secondary hypotheses are:

- The FOR technique will have a lower complication rate compared with the ACDF technique.

- The FOR technique will have lower direct and indirect costs compared with the ACDF technique.

- The FOR technique is associated with more neck pain in the first 30 days after the surgical procedure. 


\section{Trial design}

The FACET study is a nationwide, prospective, multicentre, investigator-blinded randomised controlled trial with a follow-up of 2 years. A total of 308 participants will be included in seven medical centres in the Netherlands during 2 years. Both the FOR (experimental group) and the ACDF (active control) are established surgical techniques. A non-inferiority trial design was chosen to show whether FOR has at least as much efficacy as the ACDF technique or is worse by an amount $<10 \%$ with regard to the primary outcome parameters.

\section{METHODS AND ANALYSIS}

\section{Study setting}

The study will focus on patients with a monosegmental radicular syndrome due to a lateral or foraminal herniated disc or osteophyte, with compression of the $\mathrm{C} 4$, C5, C6 or C7 nerve root. Patients will be informed about the study at the outpatient clinics of the participating hospitals. Participating neurosurgeons are from the University Medical Center Groningen, Martini Hospital Groningen, Radboud University Medical Center Nijmegen, Canisius Wilhelmina Hospital Nijmegen, Zuyderland Medical Center Heerlen, Medical Center Haaglanden and Medisch Spectrum Twente Enschede. Two centres are university teaching hospitals, and five are large regional medical centres. The participating hospitals were selected because of the high volume of spinal cases and their neurosurgeons' familiarity with the two procedures under investigation. The participating centres are evenly distributed over the Netherlands, which will result in a study population that reflects the population of the entire country.

\section{Patient inclusion criteria}

In order to be eligible to participate in this study, a participant must meet all of the following criteria:

- Age between 18 and 80 years.

- Single-level isolated cervical foraminal stenosis due to a soft disc or osteophytic component causing radiculopathy of C4, C5, C6 or C7 and requiring decompression of neuroforamen. (Foraminal stenosis due to a soft disc component is defined as: $2 / 3$ of the total discal component is located intraforaminally and a maximum of $1 / 3$ of the total discogenic component is located medially within the spinal canal. Radiculopathy is defined as pain, paresis and/or paraesthesia in corresponding nerve root distribution areas of C4, C5, C6 or C7, and must include at least arm or shoulder pain with a minimum of $30 \mathrm{~mm}$ on a $100 \mathrm{~mm}$ visual analogue scale (VAS).)

- Failure to respond to conservative treatment for 8 weeks or, during these 8 weeks, progressive signs or symptoms of nerve root compression.

- Discogenic or spondylotic foraminal stenosis (determined by MRI or CT, with or without an oblique
X-ray of the cervical spine) at the anatomical level that correlates with the clinical symptoms.

- Psychologically and physically able to comply with the study-related procedures.

- Sufficient mastery of the Dutch language to fill out the questionnaires.

- Signed and dated informed consent form, prior to any study-related procedures.

\section{Patient exclusion criteria}

A potential participant who meets any of the following criteria will be excluded from participation in this study:

- Multisegmental CRS.

- Median located disc protrusion or osteophytic protrusion (median located protrusion is defined as $>1 / 3$ of the total discogenic component is located medially within the spinal canal).

- C8 radiculopathy.

- Spinal cord compression with clinical myelopathy.

- Pure axial neck pain without radicular pain.

- Radiological signs of cervical myelopathy.

- History of cervical spine surgery.

- Morbid obesity (body mass index $>30$ ).

- Osteoporosis or chronic use of corticosteroids.

- American Society of Anesthesiologists Physical Status Classification System (ASA) 4 and 5 patients (seriously ill patients).

- Pregnancy.

- Active malignancy.

- Abundant use of alcohol, drugs or narcotics.

- Contraindications for anaesthesia or surgery.

- The participant has used another investigational drug or device within the 30 days prior to surgery.

- Incapability to speak and write the Dutch language

\section{Investigational treatment}

\section{ACDF technique:}

Microsurgical discectomy is performed by a ventral approach described by Smith and Robinson. ${ }^{6}$ The procedure can be executed with microscope or loupe magnification. The content of the intervertebral disc is exposed and removed. The posterior ligament is incised and removed with rongeurs. Disc fragments that are extruded into the neuroforamen are removed. If an osteophytic component is present, this part of the uncovertebral joint is reduced. An intervertebral spacer (cage or PMMA) is inserted into the intervertebral disc space. No additional plate fixation is used.

\section{FOR technique.}

All patients are operated in the prone position with the head in a three-point head holder. After fluoroscopy to determine the correct level, a vertical midline incision is made, and the ipsilateral lamina and facet joints are exposed. After a second confirmation of the correct level, a partial hemilaminectomy and FOR of the involved level is performed with the use of an operating microscope or loupe magnification. The amount of reduction of the facet joint is determined by the extent 
of the foraminal stenosis. In cases of soft disc compressions, the proximal root is visualised adequately and mobilised to allow the removal of the compressing disc material. However, complete removal of the soft disc component is not strictly necessary and the decision to remove the soft component will be left to the surgeon's preference. In cases of an osteophytic foraminal stenosis, bony decompression of the proximal root is performed. The surgeon has the choice to carefully coagulate and divide the venous plexus that covers the nerve sheath.

\section{Main study parameters}

The primary study parameters are 'operative success' and 'patient success'.

'Operative success' will be the postoperative decrease in radiculopathy assessed by the VAS for self-reported arm pain, between the patients operated with the FOR technique and with the ACDF technique during 24 months of follow-up. We will consider the decrease in radiculopathy clinically relevant when the postoperative decrease is more than $41 \mathrm{~mm}$ on the VAS for arm pain. $^{22}$

'Patient success' will be assessed by the modified Odom's criteria, which address physical symptoms and socioeconomic status. We will consider the first and second categories ('excellent' and 'good') as a successful outcome of the operation.

The distinction between 'patient success' and 'operative success' is made because a patient could have full improvement of the radiculopathy causing arm pain, but would not be satisfied with the operation because he or she is not able to perform his or her daily activities, for example, because of a persistent sensibility disorder in the fingertips. ${ }^{23-25}$

Secondary study parameters are:

1. Differences in postoperative work ability during 24 months of follow-up. ${ }^{26}$

2. Differences in postoperative QoL during 24 months of follow-up. ${ }^{27}$

3. Changes and differences in neck pain in the short term after the procedure (weekly assessed in the first 6 weeks postoperative) and during the 24 months of follow-up. ${ }^{23}$

4. Differences in neck disability during 24 months of follow-up. ${ }^{28}$

5. Type of complications and complication rates in the short-term (30 days) and long-term (24 months) periods.

6. Cost-effectiveness (24 months).

7. Budget impact (extrapolated to 5 years).

\section{Participant timeline}

The inclusion of participants has started on 1 December 2015. The total follow-up is 2 years. At baseline (ie, at enrolment, before the surgical procedure) and at 6, 26, 52, 78 and 104 weeks after the surgical procedure, participants will fill out web-based questionnaires. These questionnaires take $\sim 30 \mathrm{~min}$ to fill in. A short questionnaire, addressing only the arm and neck pain (VAS), will be used weekly from the surgical intervention until the visit to the outpatient clinic after 6 weeks.

The participant will visit the outpatient clinic 6 weeks after the surgical procedure, in line with standard care. An independent interviewer will contact the participants by telephone at 26, 52, 78 and 104 weeks to assess Odom's criteria. For a schematic diagram, see table 1.

\section{Sample size calculation}

The sample size analysis is based on the manuscript of Dohrmann and Hsieh. ${ }^{14}$ Based on this review of cohort studies, an overall success rate of $87 \%$ for both groups was detected. Based on the current guidelines of the CRS, there is evidence that FOR is not worse in comparison with ACDF concerning decrease in arm pain. ${ }^{2}$ Therefore, the assumption is that there will not be a statistically significant difference between the two surgical techniques concerning the primary outcome measure (relief of arm pain). With the aforementioned success rate in mind, an $\alpha$ of 0.05 , power of 0.8 and a noninferiority margin $(\delta)$ of $10 \%$, the authors conducted a sample size calculation. Including $10 \%$ drop outs, a total sample size of 308 participants was calculated, which means 154 participants per treatment arm.

\section{Recruitment}

Enrolment will take place at the outpatient clinics of the participating medical centres. When a patient with a CRS is indicated for a surgical treatment, the attending neurosurgeon judges if the patient is eligible for the trial. If the patient fulfils the inclusion and exclusion criteria, he or she is informed about the possibility to participate in the study.

To determine if the inclusion rate of 308 participants is feasible, we asked 25 patients (with a cervical radiculopathy due to a foraminal compression) in one of the outpatient clinics whether they would participate in a surgical randomised controlled trial. More than $80 \%$ of the patients were willing to participate. Furthermore, it was calculated that with a low inclusion rate of $30 \%$ each year, the authors will be able to include 308 participants in 2 years. For 7 participating centres, this implies an inclusion rate of $\sim 2$ participants per centre per month.

\section{Randomisation and treatment allocation}

Participants are randomised to either FOR or ACDF. Randomisation will be executed per participant per centre, by web-based block randomisation after the informed consent procedure is fulfilled. An independent institute, the Trial Coordination Center of the University Medical Center Groningen, will facilitate the randomisation.

\section{Blinding}

In this trial, blinding of the participant or the surgeon is not feasible. The data analysis will be performed with blinded data. Assessment of Odom's criteria will be 


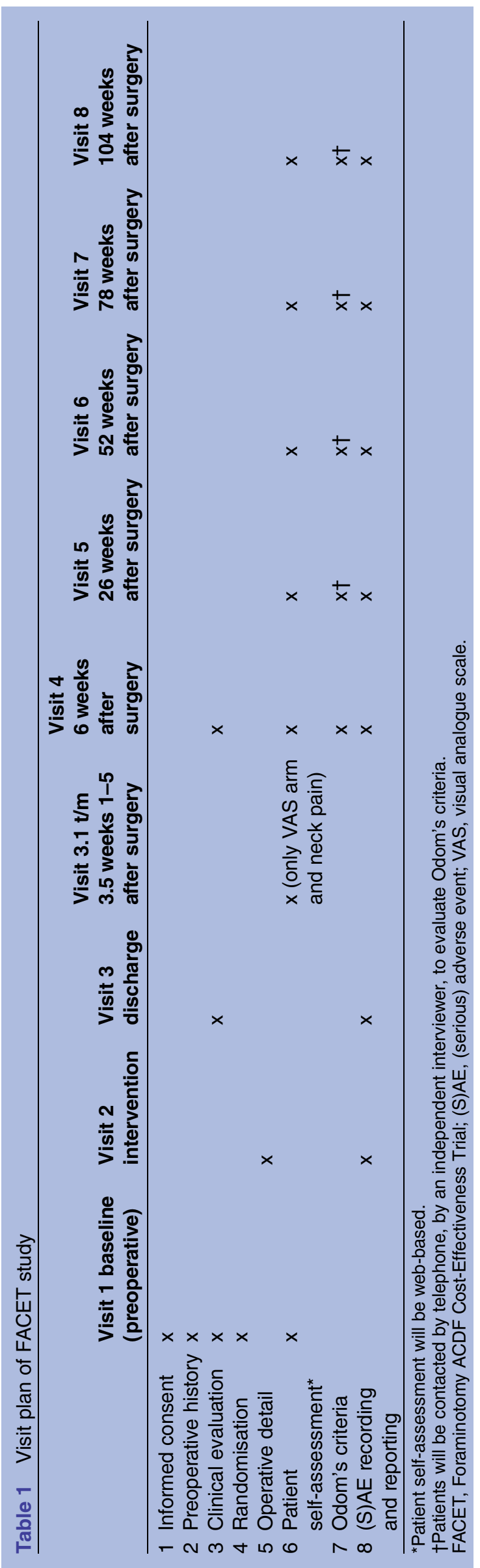

performed by an independent interviewer who is blinded to the treatment allocation.

\section{Data collection methods}

Preoperative history is obtained from standard care procedures and includes length, weight, number of months/years of neck and arm pain, signs and symptoms, other significant illnesses, pain medication (use of non-steroidal anti-inflammatory drugs) and smoking history. Information about the operative procedure will be obtained from the medical record of the participant and will include date and type of procedure, which level was operated, use of implants and the occurrence of complications during the operative procedure.

At baseline, discharge and 6 weeks after the procedure, a clinical evaluation will be performed. Information will be recorded on signs and symptoms, reflexes, sensibility and strength.

\section{Outcome measurements \\ Primary outcomes:}

'Operative success' will be the postoperative decrease in radiculopathy assessed by the VAS for self-reported arm pain, between the patients operated with the FOR technique and with the ACDF technique during 24 months of follow-up. The VAS is a well-known and validated outcome measurement. ${ }^{23}$ It is provided to the participants as a horizontal scale ranging from 0 to $100 \mathrm{~mm}$.

'Patient success' will be assessed by the modified Odom's criteria, which address physical symptoms and socioeconomic status. Odom's criteria have been widely used in studies regarding different cervical procedures and assess the improvement of the physical symptoms and the ability to perform daily activities. ${ }^{24}$ Although Odom's criteria have not been thoroughly validated yet, they have been correlated with the VAS arm pain $(\mathrm{p}=0.002)$ and VAS neck pain $(\mathrm{p}<0.0005)$. There is also a correlation between Odom's criteria and two QoL assessments for spinal disorders: the Million VAS $(p<0.0005)$ and the Oswestry Disability Index $(\mathrm{p}<0.0005) .{ }^{25}$

In the original article, Odom's criteria are defined differently for each item. Therefore, we modified Odom's criteria in analogue terms. The investigator will categorise the participant, with regard to his or her symptoms and neurological status. We will consider the first and second categories ('excellent' and 'good') as a successful outcome of the operation.

\section{Modified Odom's criteria}

1. Excellent: no symptoms referable to cervical disease. Able to perform daily activities without limitations.

2. Good: moderate symptoms referable to cervical disease. Able to perform daily activities without significant limitations.

3. Satisfactory: slight improvement of symptoms referable to cervical disease. Significant limitations in daily activities. 
4. Poor: no improvement or aggravation of symptoms referable to cervical disease. Not able to perform daily activities.

Secondary outcomes:

QoL will be assessed with the EuroQol 5 Dimensions 5 level Survey (EQ-5D-5L). This survey ranks the QoL over five dimensions in a five-level rating scale. The scores can be converted in quality-adjusted life years (QALYs). An improvement of 0.24 QALY will be considered clinically relevant. ${ }^{22}$

For neck disability, the Neck Disability Index (NDI) will be used. This questionnaire consists of 10 items, which can be ranked from 0 to 5 . The sum of these scores can be doubled to get a percentage. An improvement of $17.3 \%$ will be considered clinically relevant. ${ }^{22}$

Work ability will be assessed with the single-item Work Ability Index (WAI).$^{26} \mathrm{~A}$ participant rates his or her current ability to work, as opposed to the lifetime best ability, on a scale from 1 to 10 .

The EQ-5D-5L, NDI and single-item WAI have been tested on their validity and reliability ${ }^{26-28}$ Validated Dutch language versions are used. ${ }^{29-31}$ Neck pain is assessed with a horizontal $0-100 \mathrm{~mm}$ VAS. ${ }^{28}$ During the study, we will record the types of complications and their rates.

Cost-effectiveness and the budget impact analysis (BIA) are calculated by means of questionnaires for medical consumption and productivity costs. The National Health Care Institute of the Netherlands mentions these questionnaires as the preferred measurement tools for calculating economic evaluations. ${ }^{32}$

The questionnaires will be provided to the participant in a secure online environment. If a participant does not have access to the internet, paper questionnaires will be sent.

\section{Withdrawal of individual participants}

Participants can discontinue the participation of the study at any time for any reason. The investigator can decide to withdraw a participant from the study for urgent medical reasons (not related to the treatments under investigation).

The aim is to include 308 participants within 2 years. When a participant withdraws from the study, he or she will not be replaced since a $10 \%$ loss of inclusion is accounted for within the power calculations of the trial.

If participants withdraw after they have received the operative treatment, their permission will be asked to use the information from their medical files. Data of participants after withdrawal will be used and analysed with the intention-to-treat principle. A per-protocol analysis will be performed as well.

\section{Data management}

Data will be recorded in a web-based data capture system (OpenClinica), which is hosted by the Trial Coordination Center of the University Medical Center Groningen. This system is customised and has an audit trail facility. Ranges and programmed validation checks are implemented in the system in order to aid reliable data entry.

\section{Statistical methods}

\section{Primary study parameters}

The primary end point (postoperative decrease of selfreported arm pain (VAS)) will be defined as 'operative success'. As a second primary end point, Odom's criteria will be assessed. Odom's criteria will be defined as 'patient success'. Both items will be analysed as appropriate depending on data distribution with a one-sided 0.05 level of significance (non-inferiority). We consider the FOR technique to be non-inferior if the technique has at least as much efficacy (decrease in VAS arm pain and the good/excellent scores on Odom's criteria) as the ACDF technique or is worse by an amount $<10 \%$.

Detailed descriptive statistics will be provided for the data collected and $95 \%$ CIs will be calculated for all relevant estimates. Clinical follow-up data will be analysed by analysis of covariance or generalised model alternatives for categorical or semiquantitative data. Changes within the treatment groups over time, as well as differences between groups, will be assessed by intention-to-treat analyses. Also, the primary analysis will follow the perprotocol principle. Sensitivity analysis will be provided to evaluate robustness of the results with regard to unexpected circumstances, for example, the impact of 'crossover' and centre-related effects.

\section{Secondary study parameters}

Secondary end points will be analysed in an exploratory manner at a two-sided significance level of $5 \%$. Safety and tolerability parameters will be analysed descriptively.

Analysis of time-dependent probabilities of critical events will be performed using the Kaplan-Meier method. Furthermore, multivariate event analyses will be performed using Cox proportional hazard regression models. In addition, for the purpose of a supportive sensitivity analysis, multiple imputation procedures will be applied.

The cost-effectiveness analysis will be performed alongside the clinical trial to assess the cost-effectiveness of FOR versus ACDF. There will be two separate outcome measures for the cost-effectiveness analysis, resulting in two incremental cost-effectiveness ratios for FOR as compared with ACDF. The first is incremental costs per extra percentage of patients with arm pain relief, while the second is incremental costs per QALY gained. EQ-5D-5L scores will be converted into health state utilities using the Dutch value set. ${ }^{33}$ These health state utilities range between -0.446 and 1 , with a higher utility indicating a better health-related QoL. The utilities will be multiplied by follow-up time spent in that particular health state (area under the curve) to eventually convert into QALYs.

The analysis will be performed taking a societal perspective. The time horizon will be equivalent to the full 
follow-up of the clinical study, which is 24 months. According to pharmacoeconomic guidelines, discounting will be applied for costs $(4 \%)$ and effects $(1.5 \%)$ in the second year. ${ }^{32}$ A number of sensitivity analyses will be performed to identify the impact of variables, such as the costs of the FOR and ACDF procedures, resource use and effect size, on cost-effectiveness. A costeffectiveness acceptability curve will be constructed, based on bootstrap simulations, showing the probability of FOR being cost-effective compared with ACDF at varying levels of the willingness to pay, for either one additional percentage of patients with arm pain relief or one additional QALY.

Based on the results of the clinical study and the costeffectiveness analysis, a BIA will be performed to inform decision makers on the financial consequences of implementing FOR as treatment of first choice for cervical foraminal soft disc/osteophytic disease in the Dutch healthcare system. The BIA will be performed according to the principles of the International Society for Pharmacoeconomics and Outcomes Research (ISPOR) task force and from the perspective of the government as well as a third party payer's/healthcare insurer's perspective. ${ }^{34}$ The trial results will be extrapolated, by means of a simple model, from a time horizon of 24 months to 5 years, and for the entire Dutch population concerned. The extrapolation will assume a constant incidence of cervical foraminal soft disc/ osteophytic disease. We expect that the proportion of cases eligible for surgical intervention will be stable over time as well. Therefore, the extrapolation will be linear. Sensitivity analyses will be performed on relevant parameters such as the eventual substitution rate of FOR versus ACDF (may not be 100\%), the uptake of FOR with time, costs of the procedures and other cost items. We will assume that current usual care already consists of a mix of ACDF and FOR, that is, $90 \%$ and $10 \%$, respectively.

\section{Monitoring}

The study will be monitored by the Trial Coordination Center of the University Medical Center Groningen. This independent institute will examine the execution of the study procedures and verify source data by random sampling in the seven participating medical centres. At least one monitoring visit per year per centre will be conducted. All protocol deviations will be documented. The exact details of the monitoring will be documented in a monitoring plan in accordance with the guidelines for a low-risk to moderate-risk study.

The investigational treatments are widely accepted and frequently performed surgical procedures by neurosurgeons and orthopaedic surgeons. Participating in the study does not cause any additional risk to the participant. Therefore, the investigators decided to not establish a data monitoring committee. In the Netherlands, liability insurance is available for all participants as insurance is mandatory in the conduction of clinical trials.

\section{Box 1 List of adverse events for the FACET study}

- Death

- Thrombosis

- Pulmonary embolism

- Urinary retention

- Postoperative bleeding/haematoma

- Postoperative wound infection

- Nerve root injury

- Dural tear

- Postoperative cerebrospinal fluid leakage

- Implant malposition

- Vascular injury

- Horner's syndrome

Blood loss from the wound

- Urinary infection

- Spinal cord injury

- Oesophagus injury

- Hoarseness

- Pneumonia

- Reoperation at the index level

- Reoperation at adjacent level(s)

Other

During the complete period of the study, all adverse events will be reported. Adverse events are defined as any undesirable experience occurring to a participant during the study, whether or not considered related to intervention. The events that will be assessed are listed in box 1 .

The definition of serious adverse events is in line with the guidelines of the International Council on Harmonisation of Technical Requirements for Registration of Pharmaceuticals for Human Use (ICH) $:^{35}$

'Every adverse event will be categorised as serious if it:

- Results in death;

- Is life threatening (at the time of the event);

- Requires hospitalisation or prolongation of existing inpatients' hospitalisation;

- Results in persistent or significant disability or incapacity;

- Is a congenital anomaly or birth defect;

- Concerns any other important medical event that may not result in death, be life threatening or require hospitalisation may be considered a serious adverse experience when, based on appropriate medical judgement, the event may jeopardise the participant or may require an intervention to prevent one of the outcomes listed above'.

If a serious adverse event occurs, the local investigator is obliged to give direct notice $(<24$ hours $)$ to the sponsor. According to the Medical Research Involving Human Subjects Act, the sponsor has to report the event in $7-15$ days to the reviewing committee.

\section{ETHICS}

This study will be conducted in accordance with the Declaration of Helsinki and in compliance with the Medical Research Involving Human Subjects Act. 
Ethical approval has been granted from the Institutional Ethics Committee of the initiating medical centre on 5 November 2015.

\section{Consent}

A participant's information folder in adjusted linguistics is offered to all participants containing information about the study aims, procedures and the risks of both surgical techniques. In a conversation with the attending physician, questions regarding the study will be answered. If the patient is fully informed and willing to participate, the informed consent form is signed. The patient is provided with ample time to consider his or her decision and to ask for additional information. No maximum amount of days is set; patients can consider their decision until the surgical treatment is scheduled.

\section{Confidentiality}

The data of the participant will be recorded and analysed without any personal identifiers, by using coded information. The source documents and identification lists will be archived in a secured facility per centre. Permission for accessing data will be documented per investigator.

\section{Dissemination}

Results of this study will be disseminated through national and international papers. The participants and relevant patient support groups will be informed about the results of the study.

\section{Author affiliations}

${ }^{1}$ Department of Neurosurgery, University of Groningen, University Medical Center Groningen, Groningen, The Netherlands

${ }^{2}$ University of Groningen, University Medical Center Groningen, Groningen Spine Center, Groningen, The Netherlands

${ }^{3}$ Department of Epidemiology, University of Groningen, University Medical Center Groningen, Groningen, The Netherlands

${ }^{4}$ Radboud University Medical Center Nijmegen, Nijmegen, The Netherlands ${ }^{5}$ Department of Pharmacy, University of Groningen, University Medical Center Groningen, Groningen, The Netherlands

${ }^{6}$ Department of Neurology, University of Groningen, University Medical Center Groningen, Groningen, The Netherlands

${ }^{7}$ Department of Rehabilitation, University of Groningen, University Medical Center Groningen, Groningen, The Netherlands

${ }^{8}$ Saxion University of Applied Sciences Enschede, Enschede, The Netherlands

Acknowledgements The authors would like to thank Diane Steenks for her assistance in preparing the manuscript.

Collaborators FACET study group investigators: AEHB; JMAK; RS; GATL-L; MR Germans (Department of Neurosurgery, Radboud University Medical Center Nijmegen and Canisius Wilhelmina Hospital Nijmegen, The Netherlands); H van Santbrink (Department of Neurosurgery, Zuyderland Medical Center Heerlen, The Netherlands); N Hoess (Department of Neurosurgery, Medisch Spectrum Twente, The Netherlands); M Arts (Department of Neurosurgery, Medical Center Haaglanden, The Netherlands); J Koopmans (Department of Neurosurgery, Martini Hospital Groningen, The Netherlands); ADIvA; PCAJV; JMCVD; MFR; RJMG.

Contributors JMAK and RS initiated the study. JMAK, RS, RJMG, MFR, ADIvA, JMCVD and PCAJV designed the FACET study. JMAK, GATL-L, RS and ADIvA constructed the first draft of the protocol. AEHB and JMAK further edited the protocol conform SPIRIT guidelines. RHMAB, MRG, JK, HvS, NH and MA critically reviewed the protocol and the study procedures. All authors have given final approval for the manuscript.

Funding The study is supported by the Netherlands Organisation for Health Research and Development (ZonMW), grant number 843002604.

Competing interests None declared.

Ethics approval Medical Ethics Committee University Medical Center Groningen, 5 November 2015.

Provenance and peer review Not commissioned; externally peer reviewed.

Data sharing statement For access to the data set, a formal request should be sent to the FACET study group. The request will only be considered when the primary results of the study have been published.

Open Access This is an Open Access article distributed in accordance with the Creative Commons Attribution Non Commercial (CC BY-NC 4.0) license, which permits others to distribute, remix, adapt, build upon this work noncommercially, and license their derivative works on different terms, provided the original work is properly cited and the use is non-commercial. See: http:// creativecommons.org/licenses/by-nc/4.0/

\section{REFERENCES}

1. Radhakrishnan K, Litchy WJ, O'Fallon WM, et al. Epidemiology of cervical radiculopathy. A population-based study from Rochester, Minnesota, 1976 through 1990. Brain 1994:117:325-35.

2. Nederlandse Vereniging voor Neurochirurgie. Richtlijn behandeling van cervicaal radiculair syndroom ten gevolge van een cervicale hernia nuclei pulposi. 2010. https://richtlijnendatabase.nl/richtlijn/ cervicaal_radiculair_syndroom_tgv_chnp/conservatieve_therapie_ bij crs tgv chnp.html\#verantwoording

3. Spurling $R G$, Scoville W. Lateral rupture of cervical intervertebral disc. A common cause of shoulder and arm pain. Surg Gynecol Obstet 1944:350-8.

4. Frykholm R. Deformities of dural pouches and strictures of dural sheaths in the cervical region producing nerve-root compression; a contribution to the etiology and operative treatment of brachial neuralgia. J Neurosurg 1947;4:403-13.

5. Cloward RB. The anterior approach for removal of ruptured cervical disks. J Neurosurg 1958;15:602-17.

6. Smith GW, Robinson RA. The treatment of certain cervical-spine disorders by anterior removal of the intervertebral disc and interbody fusion. J Bone Joint Surg Am 1958;40-A:607-24.

7. Albert TJ, Murrell SE. Surgical management of cervical radiculopathy. J Am Acad Orthop Surg 1999;7:368-76.

8. Miller LE, Block JE. Safety and effectiveness of bone allografts in anterior cervical discectomy and fusion surgery. Spine 2011;36:2045-50.

9. Fehlings MG, Gray RJ. Posterior cervical foraminotomy for the treatment of cervical radiculopathy. J Neurosurg Spine 2009;10:343-4. author reply 344-6.

10. Wirth FP, Dowd GC, Sanders HF, et al. Cervical discectomy. A prospective analysis of three operative techniques. Surg Neurol 2000;53:340-6. discussion 346-8.

11. Korinth MC, Krüger A, Oertel MF, et al. Posterior foraminotomy or anterior discectomy with polymethyl methacrylate interbody stabilization for cervical soft disc disease: Results in 292 patients with monoradiculopathy. Spine (Phila Pa 1976) 2006:31:1207-14. discussion 1215-6.

12. Schebesch KM, Albert $R$, Schödel $P$, et al. A single neurosurgical center's experience of the resolution of cervical radiculopathy after dorsal foraminotomy and ventral discectomy. J Clin Neurosci 2011;18:1090-2.

13. Church EW, Halpern $\mathrm{CH}$, Faught RW, et al. Cervical laminoforaminotomy for radiculopathy: symptomatic and functional outcomes in a large cohort with long-term follow-up. Surg Neurol Int 2014;5(Suppl 15):S536-43

14. Dohrmann GJ, Hsieh JC. Long-term results of anterior versus posterior operations for herniated cervical discs: analysis of 6,000 patients. Med Princ Pract 2014;23:70-3.

15. Selvanathan SK, Beagrie C, Thomson S, et al. Anterior cervical discectomy and fusion versus posterior cervical foraminotomy in the treatment of brachialgia: the Leeds spinal unit experience (2008-2013). Acta Neurochir (Wien) 2015;157:1595-600.

16. Nanda A, Sharma M, Sonig A, et al. Surgical complications of anterior cervical diskectomy and fusion for cervical degenerative disk disease: a single surgeon's experience of 1,576 patients. World Neurosurg 2014;82:1380-7. 
17. Fountas KN, Kapsalaki EZ, Nikolakakos LG, et al. Anterior cervical discectomy and fusion associated complications. Spine 2007;32:2310-17.

18. Mansfield HE, Canar WJ, Gerard CS, et al. Single-level anterior cervical discectomy and fusion versus minimally invasive posterior cervical foraminotomy for patients with cervical radiculopathy: a cost analysis. Neurosurg Focus 2014;37:E9.

19. Jagannathan J, Sherman JH, Szabo T, et al. The posterior cervical foraminotomy in the treatment of cervical disc/osteophyte disease: a single-surgeon experience with a minimum of 5 years' clinical and radiographic follow-up. J Neurosurg Spine 2009;10:347-56.

20. Tumialán LM, Ponton RP, Gluf WM. Management of unilatera cervical radiculopathy in the military: the cost effectiveness of posterior cervical foraminotomy compared with anterior cervical discectomy and fusion. Neurosurg Focus 2010;28:E17.

21. Alvin MD, Qureshi S, Klineberg E, et al. Cervical degenerative disease: systematic review of economic analyses. Spine (Phila Pa 1976) 2014;39:S53-64

22. Parker SL, Godil SS, Shau DN, et al. Assessment of the minimum clinically important difference in pain, disability, and quality of life after anterior cervical discectomy and fusion: clinical article. J Neurosurg Spine 2013;18:154-60. JID-101223545. 0516.

23. Huskisson EC. Measurement of pain. Lancet 1974;2:1127-31.

24. Odom GL, Finney W, Woodhall B. Cervical disk lesions. J Am Med Assoc 1958;166:23-8.

25. Zoëga B, Kärrholm J, Lind B. Outcome scores in degenerative cervical disc surgery. Eur Spine J 2000;9:137-43.

26. El Fassi M, Bocquet V, Majery N, et al. Work ability assessment in a worker population: comparison and determinants of work ability index and work ability score. BMC Public Health 2013;13:305.

27. Janssen MF, Pickard AS, Golicki D, et al. Measurement properties of the EQ-5D-5L compared to the EQ-5D-3L across eight patient groups: a multi-country study. Qual Life Res 2013;22:1717-27.

28. Vernon $\mathrm{H}$, Mior $\mathrm{S}$. The neck disability index: a study of reliability and validity. J Manipulative Physiol Ther 1991;14:409-15.

29. de Zwart BC, Frings-Dresen $\mathrm{MH}$, van Duivenbooden JC. Test-retest reliability of the work ability index questionnaire. Occup Med (Lond) 2002;52:177-81.

30. Soer R, Reneman MF, Speijer BL, et al. Clinimetric properties of the EuroQol-5D in patients with chronic low back pain. Spine $J$ 2012;12:1035-9.

31. Jorritsma W, de Vries GE, Dijkstra PU, et al. Neck pain and disability scale and neck disability index: validity of Dutch language versions. Eur Spine J 2012;21:93-100.

32. Hakkaart-van Roijen L, van der Linden N, Bouwmans CAM, et al. Kostenhandleiding: Methodologie van kostenonderzoek en referentieprijzen voor economische evaluaties in de gezondheidszorg. Erasmus University Rotterdam: Institute for Medical Technology Assessment; Diemen, 2015.

33. Versteegh MM, Vermeulen KM, Evers SMAA, et al. Dutch tariff for the five-level version of EQ-5D. Value Health 2016;19:343-52.

34. Sullivan SD, Mauskopf JA, Augustovski F, et al. Budget impact analysis-principles of good practice: report of the ISPOR 2012 budget impact analysis good practice II task force. Value Health 2014;17:5-14.

35. International Conference on Harmonisation of Technical Requirements for Registration of Pharmaceuticals for Human Use. ICH harmonised tripartite guideline: Clinical safety data managment: Definitions and standards for expedited reporting E2A. Step 4 version ed. October. 1994. https://www.ich.org/fileadmin/ Public_Web_Site/ICH_Products/Guidelines/Efficacy/E2A/Step4/ E2A_Guideline.pdf 